\title{
EFFICIENCY OF \\ FAST WAVE CURRENT DRIVE FOR A WEAKLY RELATIVISTIC PLASMA
}

by

S.C. CHIU, C.F.F. KARNEY,* and Y.R. LIN-LIU

This is a preprint of a paper presented at the Tenth Topical Conference on Radio Frequency Power in Plasmas, April 1-3, 1993, Boston, Massachusetts, and to be printed in the Proceedings.

Work supported by

U.S. Department of Energy Contracts

DE-AC03-89ER51114 and DE-AC02-76CH03073

*Princeton Plasma Physics Laboratory

GENERAL ATOMICS PROJECT 3940

APRIL 1993

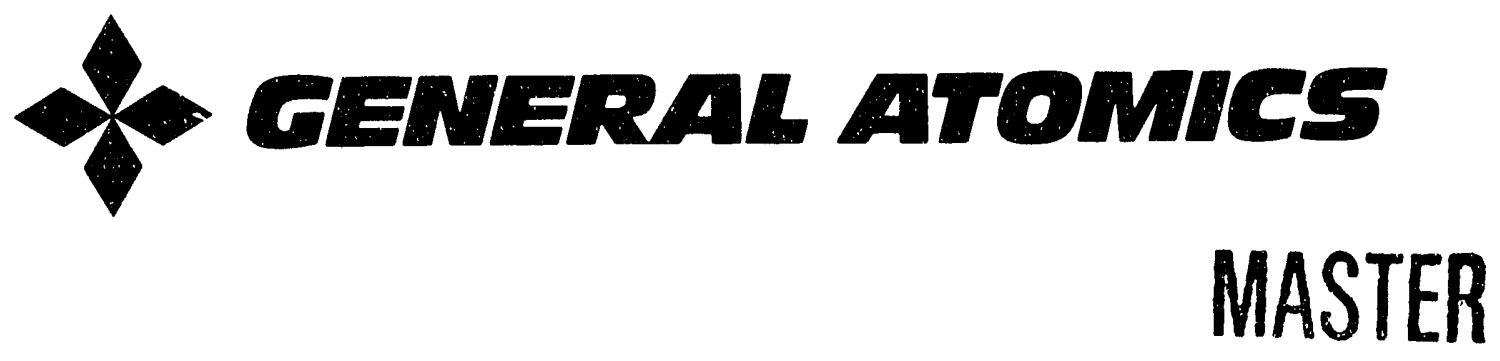




\title{
THE EFFICIENCY OF FAST WAVE CURRENT DRIVE FOR A WEAKLY RELATIVISTIC PLASMA
}

\author{
S.C. ChIU and Y.R. LIN-LIU \\ General Atomics, San Diego, CA 92186-9784 \\ C.F. F. KARNEY \\ Princeton Plasma Physics Laboratory, Princeton, NJ 08543
}

\begin{abstract}
Current drive by fast waves (FWCD) is an important candidate for steady-state operation of tokamaks. Major experiments using this scheme are being carried out on DIII-D. There has been considerable study of the theoretical efficiency of FWCD. ${ }^{1-7}$ In Refs. 4 and 5, the nonrelativistic efficiency of FWCD at arbitrary frequencies was studied. For DIII-D parameters, the results can be considerably different from the Landau and Alfven limits. At the high temperatures of reaciors and DIII-D upgrade, relativistic effects become important. In this paper, the relativistic FWCD efficiency for arbitrary frequencies is studied. Assuming that the plasma is weakly relativistic, i.e., $T_{e} / m c^{2}$ is small, an analytic expression for FWCD is obtained for high resonant energies $\left(u_{\mathrm{ph}} / u_{T_{e}} \gg 1\right)$. Comparisons with the results from a numerical code ADJ? and the nonrelativistic results ${ }^{5}$ shall be made and analytical fits in the whole range of velocities shall be presented.
\end{abstract}

\section{INTRODUCTION}

Current drive by fast waves (FWCD) is an important candidate for steady-state tokamak experiments. For this reason, there are major experiments being carried out or planned in DIII-D, Torre-Supra, JET, and other tokamaks. There was also considerable theoretical work. ${ }^{1}$ Fisch and Karney ${ }^{2}$ and Ehst and Karney ${ }^{3}$ have obtained empirical formulae for the Landau and Alfvén limits. The authors ${ }^{4,5}$ recently obtained a unified nonrelativistic formula applicable to both limits and in intermediate regimes. This is especially useful for intermediate frequencies such as in DIII-D. As the electron temperature increases to above $10 \mathrm{keV}$, relativistic effects are expected to be increasingly important. One of the authors ${ }^{6,7}$ made fully relativistic studies of the current drive efficiency in the Landau and Alfvén limits. This paper extends the work of Ref. 5 for arbitrary frequencies to include relativistic effects and extends the work of Ref. 6 to intermediate regimes. Analytic expressions for the efficiency are obtained that agree well with the numerical adjoint code ADJ. We find that in the Alfven limit the nonrelativistic efficiency formula is quite accurate even at energies when relativistic effects are separately important in absorption and current density.

\section{WEAKLY RELATIVISTIC EFFICIENCY OF FWCD}

For all practical purposes, the assumption that $\lambda^{-1}=T_{e} / m c^{2} \ll 1$ is valid, i.e., the plasma is weakly relativistic. We shall assume this in the following; nevertheless, the resonant electron energy can still be fully relativistic. 
We express momentum per unit mass $W$ in units of $V_{T_{e}}=\left(T_{e} / m c\right)^{1 / 2}$, also the efficiency $J_{1} / P_{D}$ in units of $2 e / m \nu_{0} V_{T_{e}}$ where $\nu_{0}$ is the electron collision frequency. Then

$$
\eta=J_{1} / P_{\mathrm{D}}=G\left(W_{\mathrm{p}}\right) / D_{\mathrm{A}}\left(W_{\mathrm{p}}\right)
$$

where $W_{\mathbf{p}}$ is the resonant momentum per unit mass. For a Maxwellian distribution, the absorption function $D_{\mathrm{A}}\left(W_{\mathrm{p}}\right)$ can be expressed in terms of elementary functions. For large $\lambda$ (the weakly relativistic approximation),

$$
\begin{aligned}
D_{A}\left(W_{\mathrm{p}}\right) \simeq & {\left[\frac{2}{\gamma_{\mathrm{p}}^{3}}+\frac{\alpha_{\mathrm{R}}-1}{\gamma_{\mathrm{p}}}\left(1+\frac{1}{\gamma_{\mathrm{p}}}\right)+\frac{\alpha_{2}}{\gamma_{\mathrm{p}}}\right]-\frac{1}{\lambda \gamma_{\mathrm{p}}^{2}}\left[\frac{2\left(\alpha_{\mathrm{R}}-1\right)}{\gamma_{\mathrm{p}}}+\alpha_{2}\right] } \\
& +\frac{1}{\lambda^{2} \gamma_{\mathrm{p}}^{3}}\left[\frac{6}{\gamma_{\mathrm{p}}^{2}}+\frac{6\left(\alpha_{\mathrm{R}}-1\right)}{\gamma_{\mathrm{p}}}+2 \alpha_{\mathrm{R}}\right]+O\left(\lambda^{-3}\right)
\end{aligned}
$$

where

$$
\alpha_{\mathrm{R}}+i \alpha_{\mathrm{I}}=-\frac{c^{2} \Omega_{\mathrm{e}}}{V_{T_{\mathrm{e}}}^{2} \omega} \frac{D\left(\epsilon_{33}^{\mathrm{R}}-i \epsilon_{33}^{1}\right)}{\left|\epsilon_{33}^{2}\right|\left(S-n_{n}^{2}\right)},
$$

$S, D, \epsilon_{33}$ are plasma dielectric functions, $W_{\mathrm{p}}$ is the resonant momentum, and $\alpha_{2}$ $=\left(\alpha_{\mathrm{R}}-1\right)^{2}+\alpha_{\mathrm{I}}^{2}$. One can derive analytic expressions for $G\left(W_{\mathrm{p}}\right)$ in the high $\left[G_{\mathrm{H}}\left(W_{\mathrm{p}}\right)\right]$ and low $\left[G_{\mathrm{L}}\left(W_{\mathrm{p}}\right)\right]$ energy limits. In the high energy limit, $G_{\mathrm{H}}\left(W_{\mathrm{p}}\right)$ is fairly complicated, although it is in terms of elementary functions and can be efficiently calculated. We only write down the leading order term in a $W_{\mathrm{p}}^{-1}$ series:

$$
G_{H}\left(W_{p}\right) \simeq W_{p}^{2} a_{1}\left(\gamma_{p}\right)\left[\frac{2}{\gamma_{p}^{3}}+\frac{2\left(\alpha_{R}-1\right)}{\gamma_{p}^{2}}+\frac{\alpha_{2}}{\gamma_{p}}\right],
$$

where $\gamma_{p}$ is the relativistic- $\gamma$ of resonant electrons,

$$
a_{1}\left(\gamma_{p}\right) \approx \frac{4}{\gamma_{p}^{2}\left(Z+1+4 \kappa_{1} \gamma_{p}\right)}
$$

where $\kappa_{1}=K_{1}(\lambda) / K_{2}(\lambda), K_{\mathrm{i}}$ are modified Bessel functions. On the other hand, the low energy limit is given by

where

$$
G_{L}\left(W_{\mathrm{p}}\right)=\frac{1}{W_{\mathrm{P}}}\left[\frac{1}{4} I_{4}(\infty)+\left(\alpha_{\mathrm{R}}-1\right) I_{2}(\infty)+\alpha_{2} I_{0}(\infty)\right]
$$

$$
I_{n}(\infty)=\int d W \frac{2 \pi V_{T_{e}}^{3} f_{M}}{n_{0}} \frac{\chi_{\mathrm{s}}}{\gamma^{2}} W^{n},
$$

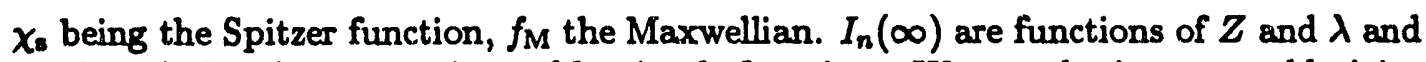
can be tabulated or approximated by simple functions. We can obtain a smoothly joint function of $G\left(W_{\mathrm{p}}\right)$ as follows:

$$
\begin{aligned}
G\left(W_{\mathrm{p}}\right)= & e^{\lambda\left(\gamma_{\mathrm{p}}-1\right)} G_{\mathrm{L}}\left(W_{\mathrm{p}}\right)+\left[1-e^{-\lambda\left(\gamma_{\mathrm{p}}-1\right)}\right] G_{\mathrm{H}}\left(W_{\mathrm{p}}\right) \\
& +\frac{W_{\mathrm{p}} e^{-\lambda\left(\gamma_{\mathrm{p}}-1\right)}}{\gamma_{\mathrm{p}}\left(Z+1+\kappa_{1} \gamma_{\mathrm{p}}\right)}\left\{\frac{A+B Z}{Z+1}+\frac{C \alpha_{\mathrm{R}}}{Z+4} W_{\mathrm{p}}^{2}\right\},
\end{aligned}
$$


where $A=11.5 \alpha_{2}+23 \alpha_{\mathrm{R}}-1.5, B=7.5 \alpha_{2}+15 \alpha_{\mathrm{R}}+4.5$, and $C=8$. There are weak temperature dependences in $A, B, C$ which can be neglected for parameters of interest. The exponential factors are suggested by the background Maxwellian.

The analytic expression $\eta_{A}=J_{n} / P_{D}$ is compared with the numerical evaluation by the adjoint code ADJ, and $\eta_{\mathrm{NR}}$ for the non-relativistic limit of $\eta$. In the relativistic calculations, the bulk temperature is taken to be $25 \mathrm{keV}$. Figure 1 is a comparison of $\eta_{A}$ with results from ADJ for various values of $Z$. The agreement is quite good. Figure 2 compares the relativistic calculations for the Alfvén $\left(\alpha_{R}=\alpha_{I}=0\right)$ and Landau $\left(\alpha_{R} \gg 1\right)$ limits at $25 \mathrm{keV}$ with non-relativistic calculations. The striking characteristic is that the non-relativistic $\eta_{\mathrm{NR}}$ appears quite accurate in the Alfvén

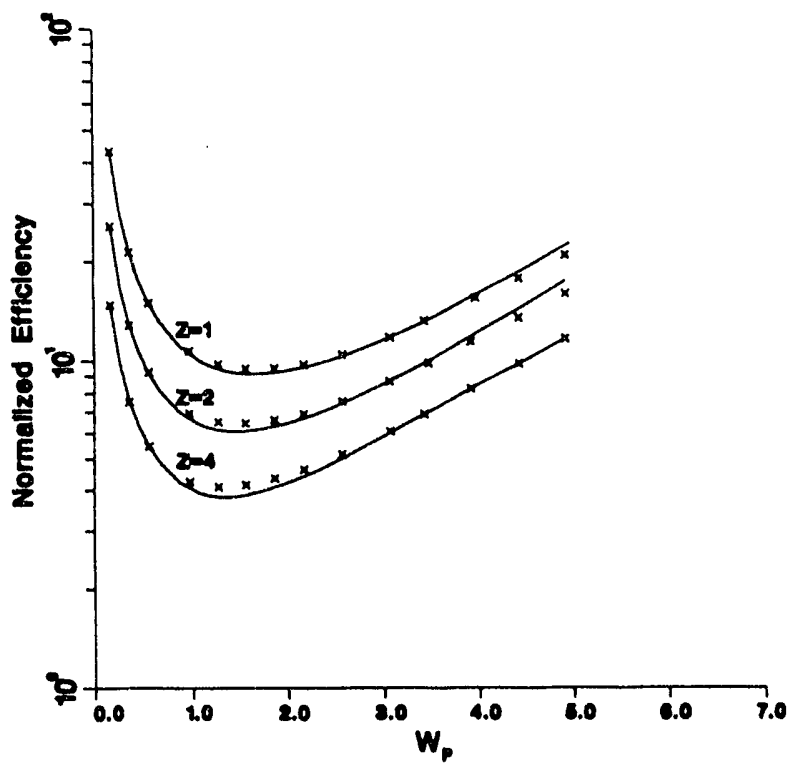

Fig. 1. Normalized $J / P$ versus resonant momentum for $1 / \lambda=T_{e} / m c^{2}=0.05$ for $Z$ $=1,2,4$. Solid lines are from ADJ code; crosses are from the analytic expression. limit even at relativistic energies $\left(\gamma_{p} \lesssim \sqrt{2}\right)$, while in the Landau limit the divergence between $\eta_{\mathrm{NR}}$ and $\eta$ (relativistic) occurs at much lower energies $(\sim 100 \mathrm{keV})$. The result at the Alfvén limit is surprising because relativity is supposed to have an effect at $\gamma$ $=\sqrt{2}$. In a closer look at the absorption (Fig. 3), we find that the relativistic effect is actually quite prominent at lower energies. Apparently, when $\alpha_{R}=\alpha_{1}=0$, the relativistic decrease in $j$, and in $P_{\mathrm{D}}$ are similar so that the ratio is rather insensitive to $\lambda$, the bulk temperature. The accuracy of Eq. (5) becomes worse as $\gamma_{p}$ becomes larger than about $\sqrt{2}$. This can be improved with a more accurate function. Results will be presented in a more detailed paper.

\section{CONCLUSIONS}

A general weakly relativistic theory of current drive is presented for parallel acceleration. The analytic expression for efficiency is found to be in good agreement with numerical results from the ADJ code. An interesting result is that the cold efficiency formula is quite accurate up to $0.5 \mathrm{MeV}$ for the Alfvén limit but less so in the Landau limit. From absorption, it is found that even in the Alfvén limit, relativistic effects set in at lower energies, but this is compensated by a similar effect on $j_{1}$ so that the ratio is not much changed.

\section{ACKNOWLEDGMENT}

This is a report of work supported by U.S. DOE Contracts DE-AC03-89ER51114 and DE-AC02-76CH03073. 


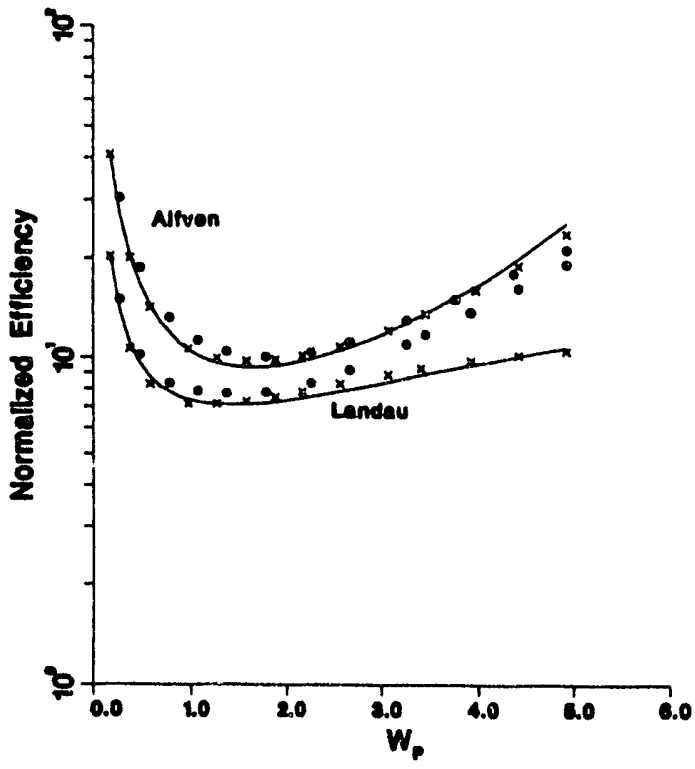

Fig. 2. Comparison between relativistic and non-relativistic $J / P$ for $Z=1$ at the Landau and Alfvén limits. Solid lines are relativistic ADJ results at $Z=1$ and $\lambda^{-1}=0.05$; crosses are from corresponding analytic expression; solid circles are non-relativistic results.

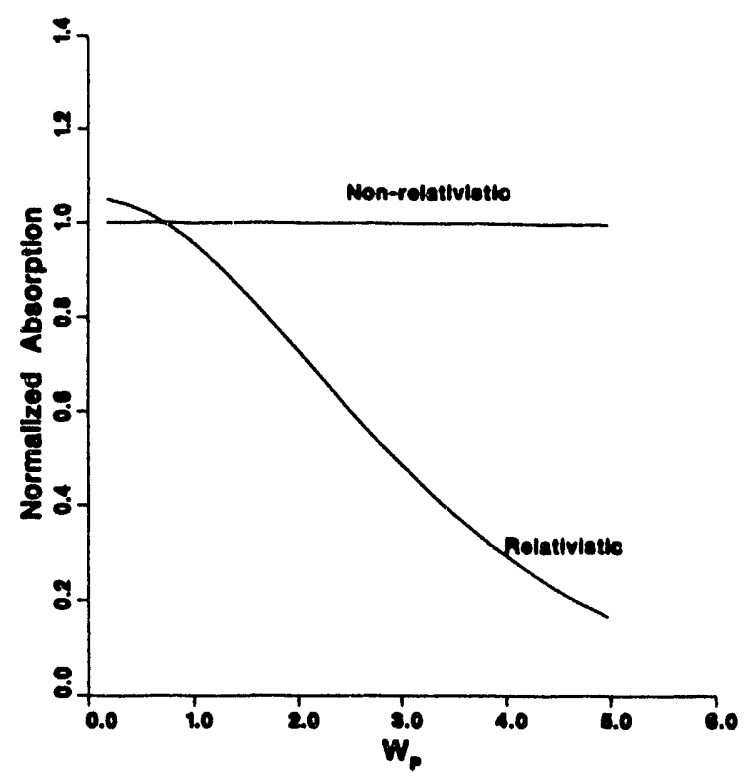

FIG. 3. Normalized absorption $D_{\mathrm{A}}$ versus $W_{\mathrm{p}}$ at the Alfvén limit.

\section{REFERENCES}

1. N.J. Fisch, Rev. Mod. Phys. 59, 175 (1987).

2. N.J. Fisch and C.F.F. Karney, Phys. Fluids 24, 27 (1981).

3. D.A. Ehst and C.F.F. Karney, Nucl. Fusion 31, 1933 (1991).

4. S.C. Chiu, V.Chan, R.W. Harvey, and M. Porkolab, Nucl. Fusion 29, 2175 (1989).

5. S.C. Chiu, C.F.F. Karney, R.W. Harvey, and T.K. Mau, in Proc. Europhysics Topical Conference on Radiofrequency Heating and Current Drive of Fusion Devices, Brussels (1992), pp. 173.

6. C.F.F. Karney and N.J. Fisch, Phys. Fluids 28, 116 (1985).

7. C.F.F. Karney, N.J. Fisch, and A.H. Reiman, in Proc. 8th Topical Conference on RF Power in Plasmas, Irvine (1989) (American Institute of Physics), p. 430. 
$\nabla$
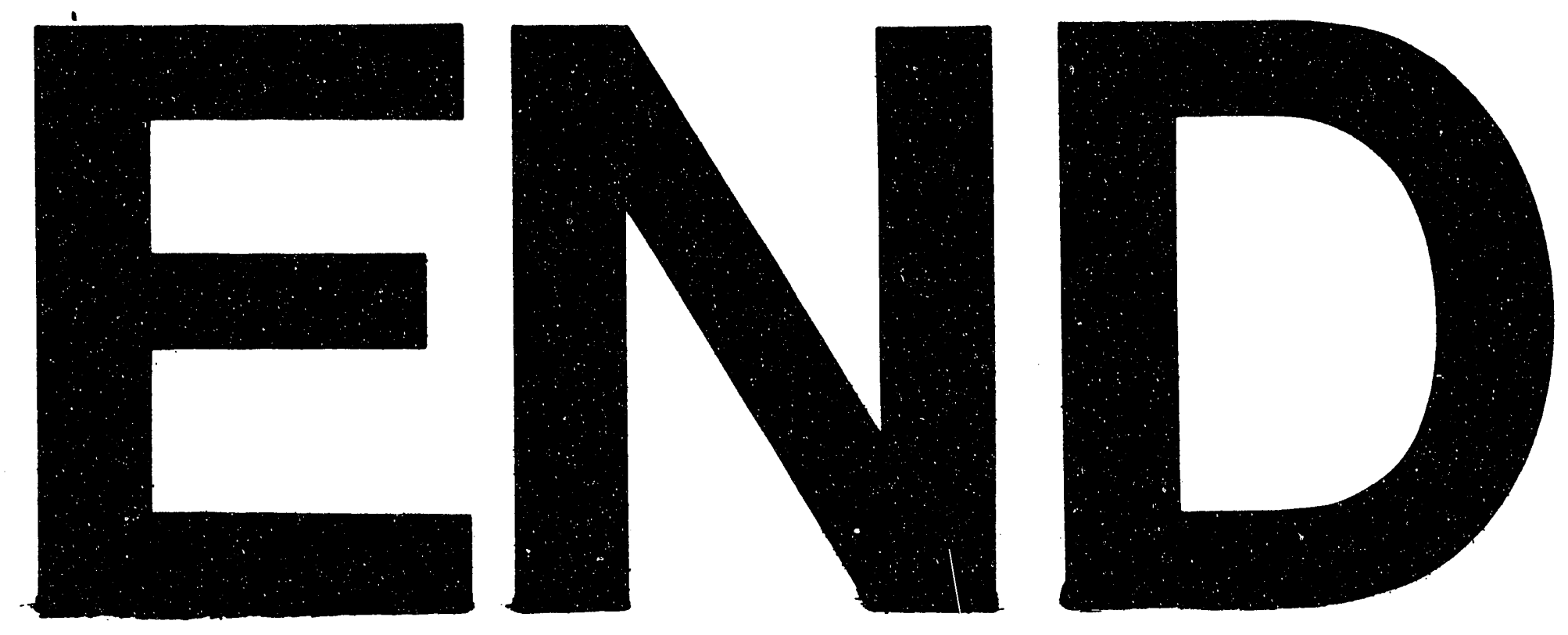

$x$
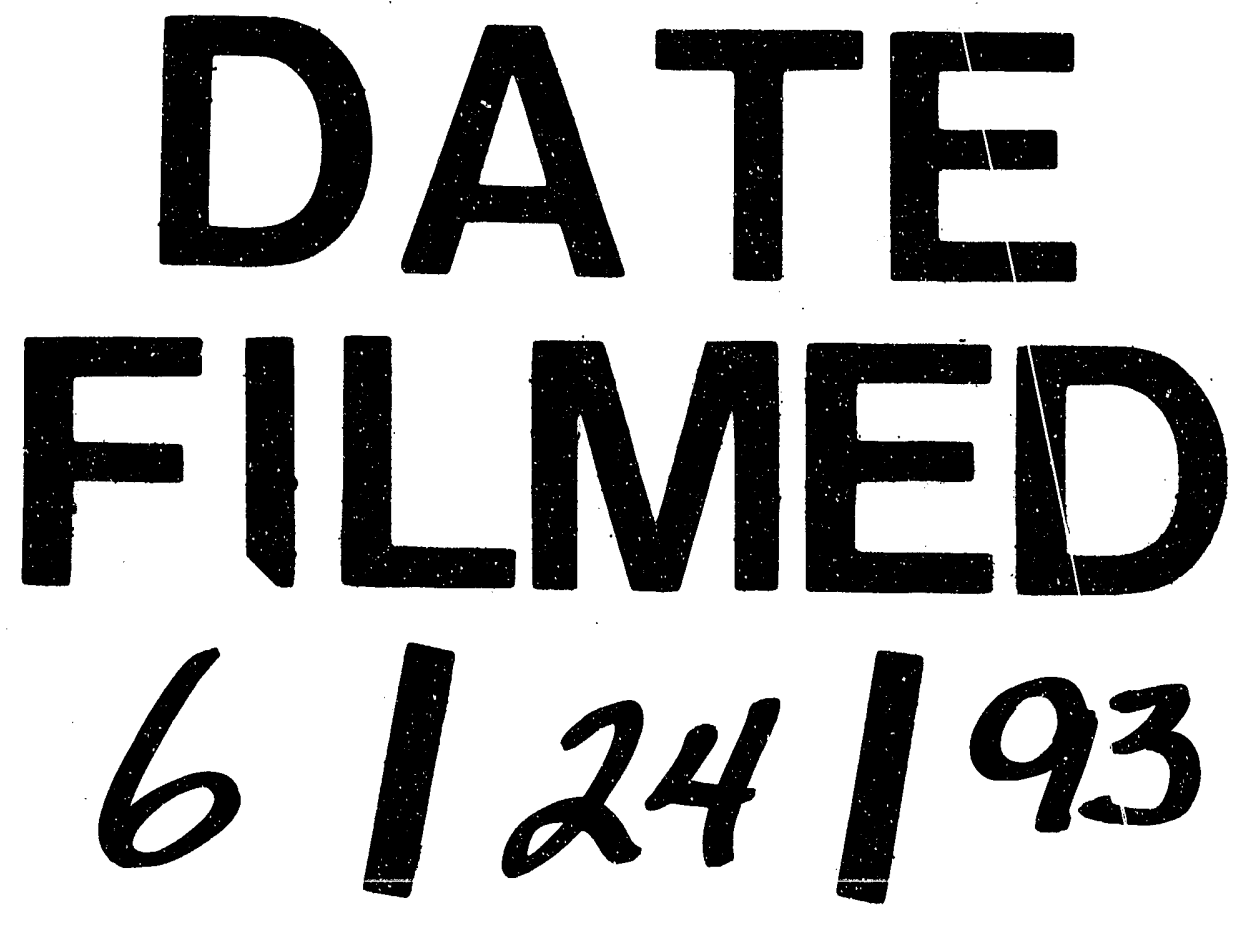
